\title{
PATRÓN DE DESARROLLO dE LA CONCIENCIA FonOLÓGICA EN NIÑOS PREESCOLARES
}

\author{
Esperanza Guarneros Reyes \\ UNAM, FES IZTACALA \\ Lizbeth O. Vega Pérez \\ UNAM, DIVISIÓN DE POSGRADO, FACULTAD DE PSICOLOGÍA CU. \\ MÉXICO
}

\section{RESUMEN}

Se identificó el patrón de desarrollo de la conciencia fonológica en niños preescolares. Participaron 302 niños de estancias infantiles públicas, el muestreo fue intencional. El diseño fue longitudinal sectorial con cohortes. Se administró la prueba Conciencia Fonológica de la Batería Evaluación de Habilidades Lingüísticas Orales y Escritas con puntuaciones de 0 a 43. Se analizó la tendencia con ajuste de curvas encontrando que los modelos matemáticos que predicen el patrón de la conciencia fonológica son los polinomiales $\left(y=-2.0297 x^{4}+22.819 x^{3}\right.$ $86.658 x^{2}+134.8 x-29.147$ ). Se concluye que el desarrollo de la conciencia fonológica tiene forma escalonada con tendencia positiva, es decir, el desarrollo no es lineal aunque incrementa con la edad, aumentan las habilidades de los niños pero se estabilizan por ciertos meses antes de volver a incrementarse. Los aumentos pueden deberse a la promoción del lenguaje oral y escrito que se hace en las escuelas de preescolar.

Palabras Clave: Patrón de desarrollo, conciencia fonológica, niños preescolares, estudios longitudinales, análisis de tendencia

\section{PATTERN OF DEVELOPMENT OF PHONEMIC AWARENESS IN PRESCHOOL CHILDREN}

\section{ABSTRACT}

This study identified the pattern of development of phonological awareness in preschool children was identified. 302 children attended public childcare facilities, sampling was intentional. The design was longitudinal cohorts sector. Phonological Awareness Test Battery Evaluation of Spoken and Written Language Skills administered with scores of 0 to 43. The trend was analyzed using curve fitting finding mathematical models that predict the pattern of phonological awareness are the polynomial $\left(y=22.819 x^{3}-2.0297 x^{4}+-134.8 x 86.658 x^{2}+-29,147\right)$. It is concluded that the development of phonological awareness has staggered positive trend, the development is not linear but increased with age, increase the skills of children but are stabilized by certain months before increasing again. The increases may be due to the promotion of oral and written language is done in preschools.

Keywords: Pattern development, phonological awareness, preschoolers, longitudinal studies, trend analysis 
BITÁCORA DEL ARTí́CULO

\section{PATRÓN DE DESARROLLO DE LA CONCIENCIA FONOLÓGICA EN NIÑOS PREESCOLARES} Esperanza Guarneros Reyes y Lizbeth $\mathrm{O}$. Vega Pérez

\section{Bitácora del Artículo:}

Recibido: 22 de septiembre de 2014

Aceptado: 27 de enero de 2015

Publicado en línea: 7 de febrero de 2015

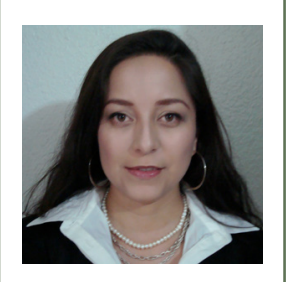

Esperanza Guarneros Reyes

UNAM - FES Iztacala

Correo: esperanzagr@gmail.com

Profesora de Carrera Asociada C Tiempo Completo de la Licenciatura de Psicología en Sistema de Universidad Abierta y Educación a Distancia SUAyED y de la Maestría en Psicología en la FES Iztacala, UNAM. Doctora en Psicología por la UNAM.

Ver más.

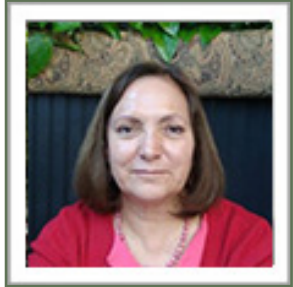

Lizbeth 0. Vega Pérez

Facultad de Psicología, División

de Posgrado, UNAM

Correo: Ivega@unam.mx

Profesora Titular B Tiempo Completo del Posgrado de la Facultad de la Psicología en la UNAM. Doctora en Psicología, 2003 por la UNAM, miembro del SNI.

Ver más...

\section{Contribución de las autoras}

Esperanza Guarneros Reyes realizó el trabajo de campo, captura, análisis de datos y redacción de manuscrito | Lizbeth O. Vega Pérez participó con la dirección del seminario para estructurar el artículo y en la redacción del manuscrito.

\section{Agradecimientos}

Este artículo se realizó gracias al financiamiento de los proyectos PAPIIT IA301915, PAPIME PE303415 y PAPIIT IT300514.

\section{Datos de filiación de las autoras}

Esperanza Guarneros Reyes | Facultad de Estudios Superiores Iztacala, UNAM. | Lizbeth O. Vega Pérez | Facultad de Psicología, División de Posgrado, UNAM. | 


\section{TABLA DE CONTENIDO}

MÉTOdO

Participantes, 74

Diseño de investigación, 74

Escenario, 74

Procedimiento, 75

Mediciones, 75

Instrumentos, 76

Análisis estadísticos, 76

RESULTADOS

CONCLUSIONES

REFERENCIAS

Meta-Análisis del Artículo

Dimensión Cuantitativa, 84

Dimensión Cualitativa, 86 
Introducción

os niños preescolares están en constante desarrollo lingüístico en el que van conociendo su mundo inmediato y su cultura, aprenden a comunicarse y comportarse dentro de un contexto social, desarrollan habilidades lingüísticas que les permiten adquirir la lectura en los años escolares y ser mejores lectores (Guarneros y Vega, 2014). Una de estas habilidades es la conciencia fonológica, que se entiende como la capacidad de reflexionar y manipular los aspectos estructurales del lenguaje hablado (Simard, Foucambert y Labelle, 2013).

Desde varias décadas atrás, la evidencia empírica muestra que la conciencia fonológica es un factor predictor de la lectura y escritura exitosas (Stanovich, 1982; Hipfner-Boucher, et al., 2014; Bus y Ljzendoord, 1999; Ehri, Nunes, Willows, Schuster, Yaghoub-Zadeh y Shanahan, 2001; Bravo-Valdivieso, 2002).También se ha encontrado que el lenguaje oral en general puede contribuir al desarrollo de la lectura temprana a través de su influencia en el desarrollo de la conciencia fonológica (Cooper, Roth, Speece y Schatschneider, 2002).

Garton y Pratt (1991) indican que el desarrollo de la conciencia fonológica inicialmente se da a través del desarrollo de la rima, como experiencia temprana que se favorece en la educación preescolar. Los niños pueden clasificar las palabras por si riman o no, esto indica una capacidad para reconocer los sonidos comunes que aparecen en algunas palabras.

La aparición en el habla de palabras que riman puede desencadenar una reflexión espontánea sobre los sonidos de las palabras. Por ejemplo, cuando se consideran las palabras monosílabas, es más fácil para los niños de preescolar atender a las partes de las palabras que son iguales (las rimas idénticas), así el niño obtiene el acceso a los fonemas individuales del inicio de las palabras.

Desde este punto, la reflexión deliberada sobre los fonemas iniciales se puede extender a otros fonemas de las palabras hasta que el niño es capaz de llevar a cabo la segmentación fonémica a un nivel que implique conciencia fonológica. Así se van desarrollando habilidades lingüísticas de la conciencia fonológica como la conciencia léxica, intrasilábica, silábica y fonémica, las cuales son habilidades lingüísticas de la conciencia fonológica (Bradley y Bryant, 1991; Herrera y Defior, 2005; Alejandro, et al., 2006; Jiménez y Ortiz, 2007).

La conciencia léxica es la habilidad de segmentar las frases en las palabras que contiene; la conciencia intrasilábica se refiere a la habilidad para segmentar las sílabas en sus componentes intrasilábicos: ataque y rima. El ataque es la parte que se constituye por la consonante o bloque de consonantes iniciales y la rima son las sílabas finales, está constituida por un núcleo vocálico y coda'; la conciencia silábica es la habilidad para segmentar, identificar o manipular conscientemente las sílabas que componen una palabra; y finalmente la conciencia fonémica es la habilidad que implica la comprensión de que las palabras están constituidas por fonemas que son unidades sonoras discretas (McBride-Chag, 2014).

Actualmente los investigadores han seguido dos enfoques metodológicos en el estudio de la conciencia fonológica: los estudios transversales y los longitudinales. Los transversales se han interesado en establecer diferencias entre la conciencia fonológica, el vocabulario y la lectura en niños sordos con implantes cocleares (Johnson y Goswami, 2010), o entre niños con y sin problemas de lenguaje (Acosta, 2013; Coloma, Cárdenas y De Barbieri, 2005). También se ha probado la efectividad de intervenciones para promover la conciencia fonológica, como el uso de una proyección dinámica sobre el conocimiento fonológico para predecir el riesgo de problemas de lectura en los niños de preescolar (Sittner y Catts, 2011), y se han comparado los métodos dinámicos y los estáticos para evaluar la conciencia fonológica (Thatcher, Wagner, Torgesen y Rashotte, 2011). En los métodos dinámicos el examinador proporciona información al alumno, por ejemplo, cuando un estudiante está teniendo dificultades en un reactivo de la prueba, el examinador puede modificar el formato, proporcionar pruebas adicionales, una estrategia útil, $u$ ofrecer una señal o indicio y los métodos estáticos o tradicionales no dan pistas a los examinados (Frenkel y Nobile, 2014).

En el caso de los estudios longitudinales, la investigación de la conciencia fonológica se ha centrado en establecer los predictores de la lectura, como la velocidad de nombrar palabras y el procesamiento fonológico a través de modelos de regresión (Aguilar, Navarro, Menacho, Alcale, Marchena y Ramiro, 2010). Los aportes de estas investigaciones han arrojado

${ }^{1}$ Coda: Es la consonante o agrupación de consonantes en posición posterior al núcleo dentro de una sílaba, es decir, después de la vocal nuclear. No es totalmente necesaria en una sílaba. Ejemplos de codas: "n" en pan, "I" en sal o "s" en es. 
hallazgos importantes. Sin embargo, han olvidado un aspecto relevante, no se encontraron estudios longitudinales que realicen un análisis del proceso de desarrollo de la conciencia fonológica en niños preescolares. A pesar de que la teoría indica que desde esa edad los niños hacen alguna manipulación de los sonidos del habla (Garton y Prat, 2001), empíricamente no se ha encontrado un modelo del patrón de desarrollo de la conciencia fonológica en los años preescolares que permita identificar los momentos en los que esta habilidad tiende a desarrollarse más, o incluso en qué momentos no se desarrolla.

Conocer cuál es el patrón de desarrollo de la conciencia fonológica en niños preescolares implicaría obtener un modelo para describir y explicar las pautas de cambio de la variable estudiada, que como señala Sidman (1978) implica encontrar las regularidades, variaciones y la dirección en el fenómeno estudiado, así como posibilitar la generalización de dichos hallazgos. Por ello el objetivo de este estudio fue identificar, describir y explicar el patrón de desarrollo de la conciencia fonológica en niños preescolares. Como hipótesis se espera que las regularidades, variaciones y la dirección del patrón de desarrollo de la conciencia fonológica estén en función de la edad de los niños preescolares.

\section{Método}

\section{Participantes}

Participaron 302 niños, 157 niñas y 145 niños, con edades entre los 3 años 6 meses y los 5 años 11 meses, que asistían a educación preescolar en estancias infantiles del sector público dentro de la Ciudad de México. La mayoría de los participantes pertenecían a familias de clase media baja con ingresos mensuales en promedio de 5 salarios mínimos y el promedio del nivel educativo de los padres fue bachillerato.

El muestreo fue no probabilístico, intencional. Dado que las unidades de análisis fueron replicativas, fue necesario homogenizar variables atributivas que la literatura demuestra influyen en el desarrollo de la conciencia fonológica, y así se cuidó la validez interna del estudio. Los criterios de inclusión en la muestra consistieron en homogenizar la muestra con base en tres variables atributivas: la escolaridad de los padres (Backhoff, Andrade, Sánchez-Moguel y Peon, 2008); las prácticas de alfabetización en casa (McBrideChag, 2014; Sénéchal y LeFevre, 2014; Sénéchal, 2012; Skibbe, Bindman, Hindman, Aram y Morrison, 2013;
Susperreguy, Strasser, Lissi y Mendive, 2007) y las prácticas alfabetizadoras en la escuela (Pataki, Metz y Pakulski, 2014; Frías, 2003; Vega, 2003, 2006, 2008).

Después de aplicar los instrumentos que evalúan estas tres variables. El criterio que se siguió para seleccionar a los niños fue que la distribución de cada variable tuvieran una simetría cercana o igual a cero ( $\mathrm{g} 1=0)$, con un sesgo entre -1 y 1 y un coeficiente de curtosis de distribución leptocúrtica (g2> 0) (Guardia, Freixa, Pero y Turbany, 2007).Lo que significa que los puntajes tuvieran poca dispersión, haciendo una distribución más elevada que la normal.

Después, los niños se asignaron a 5 cohortes por rangos de 5 meses de edad, quedando:

- Cohorte 1: niños de 3 años 6 meses a 3 años 11 meses, $\mathrm{n}=54$

- Cohorte 2: niños de 4 años a 4 años 5 meses, $\mathrm{n}=63$

- Cohorte 3: niños de 4 años 6 meses a 4 años 11 meses, $\mathrm{n}=58$

- Cohorte 4: niños de 5 años a 5 años 5 meses, $\mathrm{n}=77$

- Cohorte 5: niños de 5 años 6 meses a 5 años 11 meses, $n=50$

\section{Diseño de investigación}

El diseño de esta investigación fue un diseño longitudinal sectorial de cohortes (Glenn, 1977; Menard, 1991; Nesselroade y Baltes, 1979) y permitió mostrar las pautas de cambio en el tiempo, es decir, el patrón de la conciencia fonológica en función de la edad de niños entre 3 años 6 meses y 5 años 11 meses. Se basó en la obtención de muestras independientes para cada cohorte de edad, ya que las unidades de análisis fueron replicativas. Este tipo de diseño permite analizar los cambios en el proceso de desarrollo de una variable.

\section{Escenario}

Los directivos de las estancias facilitaron las bibliotecas y en algunos casos la oficina de la psicóloga para poder hacer la evaluación de la variable de interés. Dentro de los lugares disponibles para correr el estudio se dispuso de dos sillas pequeñas y una mesita, las sillas se colocaron haciendo ángulo de 90 grados con la mesita, de tal forma que al sentarse una investigadora 
y un niño, la investigadora tenía a un costado al niño y así le podía mostrar mejor las imágenes que contiene la prueba.

\section{Procedimiento}

El procedimiento se realizó en dos etapas:1) la selección de la muestra y 2) la obtención de los datos.

Para seleccionar la muestra se solicitó el permiso a las autoridades de estancias infantiles del D.F. y de la zona metropolitana a la Ciudad de México, obteniendo el acceso a 14 estancias infantiles. Se contó con la colaboración de directivos y maestras, quienes dieron facilidades para administrar los instrumentos de selección de la muestra.

Adicionalmente, se entregó a los padres una circular donde se explicaba el motivo de la presencia de las investigadoras y se solicitó su autorización para participar contestando el Cuestionario de actividades de lectura en el hogar (Vega, 2001), se les permitió llevárselo a casa y al regresar por los niños se les pidió que lo entregaran. Al mismo tiempo se les comunicó a las maestras de los tres grados de preescolar el objetivo del estudio y se les pidió autorización para observar sus actividades en el salón de clase para poder administrar la Lista de Cotejo de Actividades Alfabetizadoras (Vega y Rangel, 2009) y la Lista Cotejable para la Observación de Oportunidades de Desarrollo de los niños (Vega, 2008), se hicieron dos sesiones de observación en el salón de clase con duración de 30 minutos cada una, en la primera se observó el ambiente y en la segunda se registró una actividad en la que la maestra promovía el desarrollo del lenguaje y las actividades de alfabetización.

Bajo este procedimiento se obtuvieron los datos de 400 casos posibles para conformar la muestra, pero al capturar y analizar las distribuciones de las variables se descartaron los casos que quedaron fuera de los criterios de inclusión de la muestra, quedando 302 niños.

Una vez seleccionada la muestra se regresó a las estancias infantiles de los niños seleccionados y se trabajó en las bibliotecas o en la oficina de la psicóloga de la estancia infantil. La prueba de conciencia fonológica se administró de manera individual en sesiones de 30 minutos, los más pequeños de 3 años 6 meses de edad requirieron de tres sesiones para concluir la evaluación, mientras que los de 4 años 6 meses terminaron en dos sesiones y los de 5 años 6 meses y mayores terminaron, en su mayoría, en una sola sesión.

Si alguno de los niños no mostraba disposición se buscaba motivarlo invitándolo a realizar las tareas e informándole que no se le estaba calificando como bueno o malo pero que las realizara de la mejor manera posible, se le trataba con respeto y empatía; en caso de que algún niño se mostrara indispuesto no se le presionaba y se suspendía la sesión para otro día.

Una vez sentados en las sillitas la investigadora y el niño en ángulo de 90 grados y apoyándose en la mesita, la investigadora le daba las instrucciones al niño a través de un ejemplo que le permitía al niño darse cuenta de cómo debía contestar, luego el niño seguía la instrucción indicada en el ejemplo, y si el niño había comprendido la instrucción se comenzaban a realizar las tareas de la prueba. Si el niño no había comprendido, se daba otro ejemplo y se comenzaba la prueba.

\section{Mediciones}

\section{Variables}

Conciencia fonológica: Capacidad de detectar o manipular los sonidos del lenguaje oral. Implica tanto la conciencia del fonema, como la capacidad de manipular los sonidos individuales (Jason, Jeffrey, McDonald y Francis, 2006). Esta entidad está conformada por diferentes niveles, en función de la unidad lingüística objeto de reflexión y manipulación por parte del niño. Estos niveles son:

- Conciencia léxica: Es la habilidad de segmentar las frases en palabras.

- Conciencia intrasilábica: Se refiere a la habilidad de identificar si dos palabras son iguales o diferentes en las sílabas que sean ataque o en rima.

- El ataque es una parte integrante de la sílaba constituida por la consonante o bloque de consonantes inicial o intermedia de la palabra.

- La rima se forma por la vocal y consonante final de una palabra.

- Conciencia silábica: Es la habilidad de segmentar, identificar o manipular conscientemente las sílabas que componen una palabra. Ejemplo: aislar sílabas 
y fonemas, omitir sílabas y fonemas, contar sílabas y reconocer palabras descompuesta en sílabas.

- Conciencia fonémica: Es la habilidad lingüística que implica comprender que las palabras habladas están constituidas por unidades sonoras discretas, que son fonemas (McBrideChag, 2014).

\section{Instrumentos}

Se utilizó la prueba de Conciencia fonológica de la "Batería Adaptada para evaluar las Habilidades Lingüísticas Orales y Escritas de los niños preescolares" (Guarneros, 2013) producto de una investigación más amplia de la que se desprende este trabajo. Cuenta con un total de 43 ítems. La puntuación en cada ítem es de 1 punto si es correcta la respuesta y 0 si es incorrecta. La administración es individual. La confiabilidad es de .90. La prueba contiene cuatro sub pruebas:

Segmentación léxica: Consta de oraciones de dos y tres palabras, que representan situaciones cotidianas que se presentan de manera verbal. El niño debe reconocer el número de palabras que están contenidas en la oración ayudándose de los dedos de la mano o de palmadas para contarlas.

Conciencia intrasilábica: El niño identifica las palabras que suenan igual o diferente, al final cuando riman y al principio cuando es ataque.

\section{Conciencia silábica:}

A) Aislar sílabas y fonemas: Consiste en buscar en series de dibujos, los que en su nombre contengan: 1) El fonema vocálico emitido por el examinador en posición inicial y final; 2) la sílaba en posición inicial y final, y 3) el fonema consonante en posición inicial y final.

B) Omisión de sílabas y fonemas en las palabras: Consiste en nombrar series de dibujos omitiendo: primero, el fonema vocálico inicial; segundo, la sílaba inicial, y tercero, la sílaba final. En esta tarea se omiten fonema vocálico, fonema consonante y sílabas.

C) Contar las sílabas en una palabra: Implica que el niño diga cuántas partes tiene una palabra, debe segmentarlas en sílabas.

D) Síntesis: reconocer y pronunciar la palabra descompuesta en una secuencia de sílabas:
Se le dicen de forma oral al niño palabras separadas en sílabas y él debe reconocer qué palabras son.

Conciencia fonémica: Contiene tareas de síntesis y de segmentación fonémicas:

A) Síntesis: En esta tarea se dicen los sonidos de las letras que forman una palabra y se le pide al niño diga qué palabras forman.

B) Segmentación: Es la tarea inversa a la anterior, se le dice una palabra y se espera que el niño diga por qué fonemas está compuesta.

\section{Análisis estadísticos}

En el análisis se obtuvo el porcentaje promedio de respuestas correctas en la variable y en sus componentes conciencia léxica, conciencia silábica, conciencia intrasilábica y conciencia fonémica por cada cohorte de edad. De esta manera el $100 \%$ significa haber obtenido la calificación máxima en una prueba.

Una vez graficado el patrón de desarrollo de la conciencia fonológica, se realizó el análisis de tendencia por ajuste de curvas lineales y no lineales (Silva, 2004), ya que mediante este análisis se pueden encontrar los patrones o modelos que explican las regularidades, variaciones y la dirección de la conciencia fonológica, así como posibilitar la generalización de dichos hallazgos (Sidman, 1978).

Para seleccionar el modelo que explica el patrón de desarrollo de la conciencia fonológica y sus componentes se buscó que el coeficiente de determinación de la recta $\left(R^{2}\right)$ fuera cercano a 1.

\section{Resultados}

El modelo que mejor explica el patrón de desarrollo de la conciencia fonológica con sus componentes conciencia léxica, intrasilábica, silábica y fonémica fue el modelo polinomial de cuarto grado, ya que se obtuvo un $\mathrm{R}^{2}$ cercano a 1 o igual a 1 . (Ver Tabla1).

En general, los modelos matemáticos identificados significan que las habilidades de la conciencia fonológica se desarrollan en los niños preescolares con una tendencia positiva de forma polinomial de cuarto grado, es decir, de manera curvilínea con variabilidad y estabilidad entre las puntuaciones cada 5 meses de edad. Los aumentos se aprecian con etapas de cima y de valle, esto indica que las habilidades de conciencia 
Tabla 1

Modelos polinomiales encontrados para los componentes de la Conciencia fonológica

\begin{tabular}{|r|c|c|}
\hline $\begin{array}{c}\text { Componentes de la } \\
\text { Conciencia } \\
\text { Fonológica }\end{array}$ & Ajuste polinomial & $\mathbf{R}^{\mathbf{2}}$ \\
\hline $\begin{array}{r}\text { Conciencia léxica } \\
\text { Conciencia }\end{array}$ & $\mathrm{y}=-3.8754 \mathrm{x}^{4}+45.351 \mathrm{x}^{3}-181.52 \mathrm{x}^{2}+295.54 \mathrm{x}-112.17$ & $\mathrm{R}^{2}=1$ \\
\hline $\begin{array}{r}\text { intrasilábica } \\
\text { Conciencia silábica }\end{array}$ & $\mathrm{y}=-1.9898 \mathrm{x}^{4}-4.9445 \mathrm{x}^{3}+24.669 \mathrm{x}^{2}-42.183 \mathrm{x}+66.575$ & \\
\hline $\begin{array}{r}\text { Conciencia } \\
\text { fonémica }\end{array}$ & $\mathrm{y}=-3.0304 \mathrm{x}^{4}+34.359 \mathrm{x}^{3}-131.64 \mathrm{x}^{2}+201.47 \mathrm{x}-76.904$ & $\mathrm{R}^{2}=0.973$ \\
\hline $\begin{array}{r}\text { CONCIENCIA } \\
\text { FONOLÓGICA }\end{array}$ & $\mathrm{y}=-2.0297 \mathrm{x}^{4}+22.819 \mathrm{x}^{3}-86.658 \mathrm{x}^{2}+134.8 \mathrm{x}-29.147$ & 1 \\
\hline
\end{tabular}

fonológica incrementan, alcanzan una cima, luego se mantienen sin incrementos considerables, más tarde vuelven a aumentar y llegan a otra cima (Ver Figura1).

De manera particular los resultados indican en el caso de la conciencia léxica, que consiste en la habilidad de identificar cuántas partes tiene una frase; que tiene un patrón que muestra que cuando los niños tienen 3 años con 6 meses tienen un desarrollo de esta habilidad del $43.33 \%$, y al pasar a los 4 años habilidad para segmentar frases verbalmente aumenta un 10\%; cuando tienen 4 años 6 meses se mantiene sin incrementos y a los 5 años la habilidad aumenta un $20 \%$ más, alcanzando un nivel de desarrollo del $76.10 \%$, el cual a los 5 años 6 meses se mantiene. Cabe señalar que todos los niños prefirieron segmentar las oraciones ayudándose de las palmas.
De manera descriptiva, en cuanto a la conciencia léxica, los resultados muestran que los niños preescolares presentan la habilidad de manipular las frases segmentándolas en palabras en diferentes grados; los niños de 3 años 6 meses de edad sólo en algunas ocasiones logran segmentar frases de dos palabras, por lo que no se puede afirmar que posean esta habilidad. Los niños de 4 años hasta la siguiente cohorte de 4 años 11 meses, sí son capaces de segmentar frases pero sólo cuando se componen de dos palabras y con ayuda de sus palmas. A partir de los 5 años en adelante cuentan con la habilidad de manipular una frase segmentándola en el número de palabras que la componen, cuando éstas tienen dos o tres palabras; incluso los niños de 5 años 11 meses lo pueden realizar sin ayuda de las palmas.
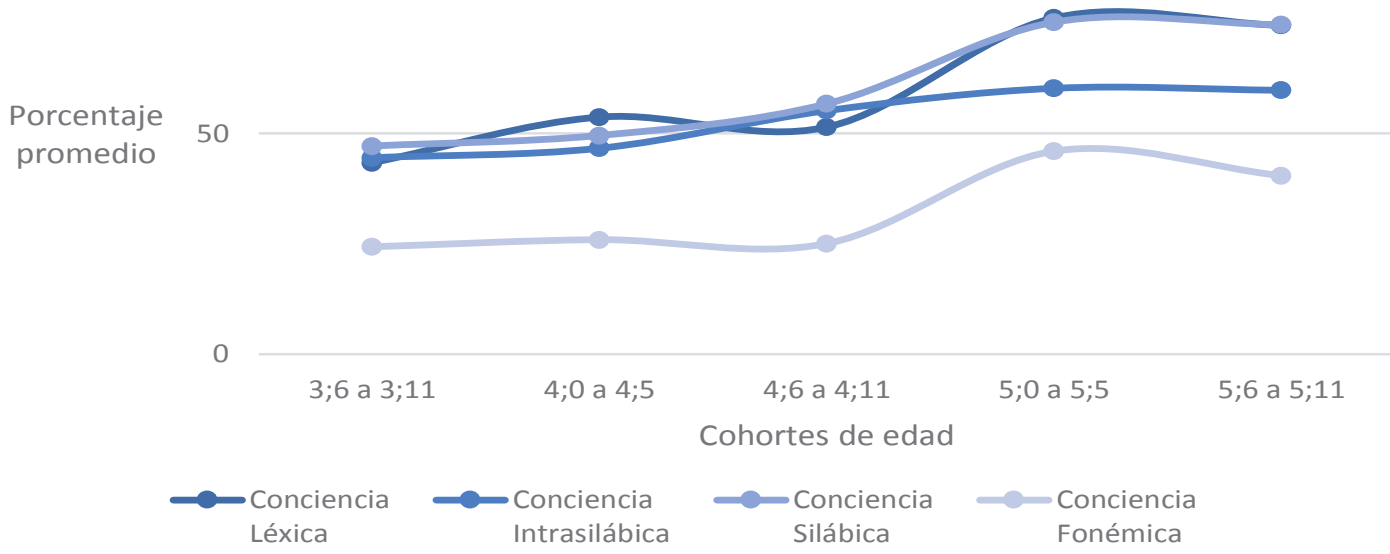

Figura 1

Patrón de desarrollo de las habilidades que comprenden la conciencia fonológica 
Sobre la conciencia intrasilábica, en la que los niños tienen que reconocer qué palabras suenan igual o diferente al final de la palabra para el caso de la rima y al principio en caso del ataque, los resultados indican que a los 3 años 6 meses los niños cuentan con un $44.4 \%$ de conciencia intrasilábica, a los 4 años aumentan sólo un 2\%, pero a los 4 años con 6 meses incrementa la habilidad un $10 \%$. Éste es el momento en el que hay más incremento, sube $5 \%$ a los 5 años de edad y ahí se mantiene hasta los 5 años 11 meses.

Esto quiere decir que aunque la conciencia intrasilábica va incrementándose, los niños no logran los niveles que sí alcanzan en la conciencia léxica. En términos descriptivos se encontró que a los niños de 3 años 6 meses les cuesta trabajo identificar la rima, y de los 4 años hasta los 5 años 11 meses sí la identifican, y los niños de 5 años en adelante no sólo aciertan en todos los casos a identificar si dos palabras riman, también pueden expresar cuál es la parte de las dos palabras que rima. Por ejemplo, pueden decir que "pato" y "plato" riman y que son similares en "ato".

Respecto al ataque, los resultados señalan que identificar si dos palabras suenan similar es una habilidad más difícil para los niños más pequeños, ya que tanto los niños de 3 años 6 meses de edad como los de 4 años de edad no la poseen; es hasta los 4 años 6 meses de edad que pueden realizarlo, pero aun los niños de 5 años con 11 meses tuvieron errores, lo que indica que no es una habilidad que se tenga desarrollada completamente, ya que en ocasiones señalaron igualdad o diferencia, pero no pudieron expresar en qué consistían éstas, por ejemplo: al pedirles que dijeran si carro y carreta era iguales o diferentes, los niños más pequeños pueden decir que son iguales pero no por qué, ni indicar que son iguales en "carr".

En la conciencia silábica, que consiste en aislar las sílabas y fonemas, omitir sílabas y fonemas en las palabras, contar las sílabas en una palabra y finalmente reconocer y pronunciar la palabra descompuesta en una secuencia de sílabas, se encontró que los niños de 3 años 6 meses logran un $47.11 \%$ de esta habilidad, manteniéndose con cierta constancia a los 4 años; a los 4 años 6 meses incrementa la conciencia silábica hasta que a los 5 años de edad los niños alcanzan un nivel del $75.16 \%$ logrando aislar y omitir sílabas y fonemas, contar las sílabas de una palabra y descomponer en una secuencia de sílabas las palabras.

En términos descriptivos esto quiere decir que el desarrollo de la conciencia silábica se presentó de la siguiente manera:

- Los niños de 3 años 6 meses logran aislar sílabas o fonemas del principio de palabras que iniciaban con vocal, omitir sílabas y fonemas al final de palabras monosílabas.

- Los niños de 4 años en adelante pueden decir el nombre de un dibujo omitiendo el final, pero no pueden hacerlo si se les pide omitir el principio de una palabra; tampoco consiguen contar las sílabas en una palabra, ni reconocer y pronunciar la palabra descompuesta en una secuencia de sílabas.

- Los niños de 4 años 6 meses pueden aislar fonemas del inicio de las palabras sin importar si eran vocales o consonantes, pero no aislar fonemas y sílabas del final de las palabras; logran contar las sílabas en una palabra ocasionalmente con palabras de dos sílabas, y pronunciar una palabra segmentándola en sílabas.

- Los niños de 5 años en adelante aíslan fonemas y sílabas al principio y final de las palabras; cuentan palabras de dos y tres sílabas, en partes segmentadas y con ayuda de sus palmas, y pronuncian la palabra descompuesta en una secuencia de sílabas.

La cuarta y última habilidad de conciencia fonológica fue la conciencia fonémica, que consistió en que los niños pudieran decir qué palabra se forma con ciertos sonidos y que pudieran descubrir los fonemas de las letras que conformaban una palabra. Se encontró que el desarrollo de esta habilidad es menor en comparación con el resto de los componentes de la conciencia fonológica. Se observó a primera vista que es una habilidad difícil para los niños, ya que en todas las cohortes obtuvieron puntuaciones menores en comparación a las puntuaciones en otras habilidades. De los 3 años 6 meses a los 4 años con 11 meses los niños obtuvieron un $25 \%$ en promedio de la habilidad, fue hasta los 5 años que los niños incrementaron esta habilidad al 45\%, logrando identificar las palabras al presentárseles sus sonidos sólo cuando las palabras tenían tres sonidos, (por ejemplo s-o-l= sol); sin embargo, les fue muy difícil hacerlo con palabras de cuatro y cinco sonidos (ejemplo m-e-s-a= mesa). Cuando se trató de separar una palabra por el sonido de sus fonemas, se observó un mayor nivel de dificultad, ya que sólo los niños de 5 años a 5 años 11 meses lograron separar palabras por sus fonemas en 
los casos de palabras con tres sonidos y donde dos de ellos eran vocales (ejemplo Oso= o-s-o), sin embargo, ningún niño pudo acertar en las palabras de cuatro sonidos. Esto indica que identificar a qué palabra corresponden los sonidos de una serie de fonemas, o descomponer una palabra en sus fonemas, es la habilidad más compleja de la conciencia fonológica y su mayor desarrollo se alcanza después de los 5 años de edad, aun así, los niños de esta edad no alcanzan a demostrar ni un $50 \%$ de la habilidad.

En resumen, se logró identificar el patrón de desarrollo de la conciencia fonológica en niños preescolares, este patrón sigue un modelo polinomial de tendencia positiva, explicado con la ecuación: $y=-2.0297 x^{4}+$ $22.819 x^{3}-86.658 x^{2}+134.8 x-29.147$

Los resultados permiten afirmar que las habilidades de conciencia fonológica de los niños preescolares van aumentando según la edad de los niños, pero no de forma lineal sino que existen momentos en los que se dan incrementos poco notables y otros muy acelerados, por lo que las pautas de cambio que el estudio logró identificar y describir son:

- Cohorte 1: Entre los 3 años 6 meses y los 3 años 11 meses ya existen indicios de conciencia fonológica entre el $24.26 \%$ y $44.44 \%$ de su desarrollo.

- Cohorte 2: De los 4 años a los 4 años 5 meses aumenta el desarrollo entre un $25.87 \%$ y un $49.50 \%$.

- Cohorte 3: Entre los 4 años 6 meses y los 4 años 11 meses la conciencia fonológica incrementa entre un $25 \%$ y $55.17 \%$. Aunque en conciencia léxica y fonémica desciende, en conciencia intrasilábica y silábica el niño sigue mejorando sus habilidades, aumentando sus puntuaciones.

- Cohorte 4: Entre los 5 años a los 5 años 5 meses el incremento es considerable entre el $45.97 \%$ y el $76.10 \%$. Esenestaedadenlaquetodaslashabilidades se aceleran en su desarrollo, siendo el cambio mayor en latendencia del patrón dela conciencia fonológica.

- Cohorte 5: De los 5 años 6 meses a los 5 años 11 meses se mantiene entre un $40.40 \%$ y un $74.55 \%$, por lo que se muestra un descenso en todas las habilidades.

Los incrementos de las diferentes habilidades de la conciencia fonológica y su forma son explicados por la ecuación encontrada:

$y=-2.0297 x^{4}+22.819 x^{3}-86.658 x^{2}+134.8 x-29.147$

La cual es una curva de desarrollo que da cuenta de la relación funcional entre la edad y la conciencia fonológica.

\section{Conclusiones}

Los resultados de este estudio permitieron identificar un modelo matemático que describe el patrón de desarrollo de la conciencia fonológica en niños preescolares y que al mismo tiempo puede explicarlo, ya que la ecuación encontrada es un modelo de regresión que permite obtener el desarrollo de conciencia fonológica que se espera tenga un niño preescolar en determinada edad, por lo tanto se puede asegurar que el objetivo se cumplió.

Así mismo, se puede afirmar que las habilidades de la conciencia fonológica de los niños preescolares van aumentando según la edad de éstos con un patrón polinomial, es decir, aumenta con incrementos variables, con cimas y valles; este hallazgo empírico confirma que el desarrollo de la conciencia fonológica no es lineal (Gillam, Gillam y Reece, 2012) y no sólo eso, sino que los resultados muestran la topografía del desarrollo de la conciencia fonológica.

Dicha tipografía permite observar varios indicadores como el nivel de conciencia fonológica con el que parten los niños preescolares, e identificar las edades relevantes donde se dan los incrementos acelerados en el desarrollo de las habilidades analizadas, permite conocer los cambios que se dan en la variable según la edad de los niños.

En cuanto al nivel de inicio de desarrollo de la variable estudiada se encontró que los niños preescolares de 3 años 6 meses de edad mostraron puntuaciones diferentes de cero en todas las tareas de las habilidades de conciencia fonológica sin haber tenido instrucción alguna al respecto (conciencia léxica $43.33 \%$, conciencia intrasilábica $44.44 \%$, conciencia silábica, $47.11 \%$ y conciencia fonémica $24.25 \%$ ), esto es consistente con los hallazgos Hipfner-Boucher, et al. (2014) y de Anthony y Francis (2005), quienes encontraron que los niños de 3 años de edad presentaban sensibilidad fonológica en todos los niveles de complejidad lingüística, lo que pone de manifiesto que los niños no necesariamente 
deben dominar una habilidad menos compleja antes de dominar la siguiente de mayor complejidad. Este resultado se observó en todas las cohortes de edad. Desde los niños más pequeños hasta los más grandes lograron realizar algún grado de las tareas de todas las habilidades de conciencia fonológica evaluadas, permitiendo afirmar que las habilidades de conciencia fonológica se desarrollan a lo largo de un continuo (Vance, Stackhouse y Wells, 2005).

Sobre las edades relevantes donde se dan los incrementos acelerados en el desarrollo de las habilidades analizadas, se encontró que el desarrollo de las habilidades de la conciencia fonológica se aceleró en los grupos de mayor edad, esto es consistente con el estudio de Hipfner-Boucher, et al. (2014). Sin embargo, a diferencia de los resultados de estos autores los aumentos sustanciales no se dieron entre los 3 y los 4 años; en este caso se dieron en los niños entre los 5 años y los 5 años 5 meses de edad. Esto puede deberse particularmente a las características educativas de la muestra de este estudio, ya que los niños de 5 años de edad en adelante se encuentran cursando el tercer grado de preescolar, que es el grado en donde se empiezan a realizar mayores actividades explícitas de desarrollo del lenguaje oral y sobre todo del lenguaje escrito. Aunque no se busca que los niños aprendan a leer y escribir convencionalmente, las actividades que promueven las maestras con los niños utilizan el abecedario recortando las letras de su nombre, del de mamá o de sus amigos, empiezan a identificar el nombre de las letras y sus sonidos. De esta manera se van enseñando las letras, lo cual es una variable que la literatura ha señalado se correlaciona con el aumento de la conciencia fonológica (Kim, Petscher, Foorman y Zhou, 2010).

Asimismo, en el caso particular de la conciencia fonémica, se observó que en todas las cohortes de edad, los niños obtuvieron puntuaciones inferiores en comparación a otras tareas como las de segmentar las frases en palabras (conciencia léxica) o las de aislar sílabas y fonemas, omitir sílabas y fonemas, contar sílabas y reconocer palabras descompuestas en sílabas (conciencia silábica); hasta los 5 años de edad se observó un incremento mayor. Esto puede explicarse porque las actividades del tercer grado de preescolar implican tareas de alfabetización. En estas actividades los niños tienen experiencias con el lenguaje escrito y esto influye dramáticamente en el desarrollo de la conciencia fonológica, en especial el desarrollo de la conciencia fonémica.

Como señalan Lonigan, Schatschneider y Westberg
(2008) la mayoría de los niños alcanzan niveles mínimos del conocimiento de los fonemas antes de la alfabetización; cuando aprenden los nombres de las letras y sus sonidos acceden a los fonemas, y como los fonemas producidos en el habla son acústicamente inseparables de las letras, la conciencia fonológica se desarrolla rápidamente. Una vez que la instrucción en la lectura y la escritura comienzan, se mantiene una relación recíproca entre el aprendizaje de la lectura y el desarrollo de la conciencia fonológica.

Finalmente como contribución y directriz futura de esta investigación, se considera que la contribución principal de este trabajo tiene una implicación teórica y metodológica.

La implicación teórica consiste en la demostración empírica de que el desarrollo de la conciencia fonológica no es lineal; esto quiere decir que el desarrollo de la conciencia fonológica desde la linealidad guardaría una relación de proporcionalidad entre una edad anterior y una edad posterior, pero en una concepción del desarrollo por los resultados de este estudio, se concibe como no lineal, esto implica que las relaciones de la edad de los niños con el desarrollo de la conciencia fonológica y sus respectivas habilidades que la componen es de carácter iterativo, que muestra una inestabilidad la cual da cuenta de los cambios en el desarrollo.

A nivel metodológico, el abordaje con visión longitudinal realizado para identificar, describir y explicar el patrón de desarrollo, permitió el análisis del proceso con sus cambios más importantes en la edad en la que la conciencia fonológica se vuelve indispensable para la adquisición de la lectura en edad escolar; a diferencia de cuando se sigue un diseño longitudinal, únicamente para establecer el nivel de predicción de la conciencia fonológica en el aprendizaje de la lectura y escritura convencionales.

Además, este patrón, encontrado en los niños de clase media-baja, dezonasurbanas, con padres de escolaridad entre bachillerato y estudios de licenciatura; puede utilizarse para detectar el nivel de desarrollo esperado en un niño con estas características, o incluso como un modelo de comparación con niños que difieran de estas características.

A partir del patrón de desarrollo encontrado fue posible mostrar que desde los 3 años 6 meses hay cierto desarrollo de las habilidades de conciencia fonológica y que a los 5 años se dan los incrementos más acelerados, se propone analizar si los patrones de 
desarrollo aquí encontrados difieren con los patrones de desarrollo en los que a los niños más pequeños se les involucre más en actividades de lenguaje escrito.

Esta posibilidad de comparar los patrones de desarrollo de conciencia fonológica con los de otros niños que tengan diferente desarrollo en el lenguaje escrito o bien que difieran en variables atributivas, ayudaría a establecer regularidades más consistentes y explicaciones teóricas más amplias.

Para concluir, los resultados encontrados y el abordaje metodológico que tuvo este estudio aportan datos empíricos para la psicología, que permiten analizar la lectura y la escritura en niños pequeños, como un fenómeno interactivo a la manera de un proceso de desarrollo humano que evoluciona escalonadamente, es decir, por momentos con incrementos acelerados y en otros se mantiene estable en el tiempo.

\section{Referencias}

Acosta R. V. (2012). Algunos retos y propuestas en la conceptualización, evaluación e intervención del Trastorno Específico del Lenguaje (TEL). Revista Chilena de Fonoaudiología * ISSN 0717-4659, 11(0), 23-36.

Aguilar V., Navarro G., Menacho, J., Alcale, C., Marchena, C. y Ramiro, O. (2010). Velocidad de nombrar y conciencia fonológica en el aprendizaje inicial de la lectura. Psicothema, 22(3), 436-442.

Anthony, J. L., \& Francis, D.J. (2005). Development of Phonological Awareness. Current directions in psychological science. American Psychological Society, 14(5), 255-259.

Backhoff, E., Andrade, E. Sánchez-Moguel, A. \& Peon, M. (2008). El Aprendizaje en tercero de preescolar en México. México: Instituto Nacional para la Evaluación del Educación.

Frenkel, S. \& Nobile, D. (2014). Learning to learn:Assessment of metacognitive competencies. 8th International Technology, Education and Development Conference INTED2014.SimposiocelebradoenValencia, España.

Gillam, S. L., Gillam, R. B. \& Reece, K. (2012). Language outcomes of contextualized and decontextualized language intervention: Results of an early efficacy study. Language, speech, and hearing services in schools, 43(3), 276291.

Glenn, N. (1977). Cohort analysis. Beverly Hills, CA: Sage.

Guardia, J., Freixa, M. Peró, M. \& Turbany, J. (2007). Análisis de datos en Psicología. España: Delta.
Guarneros, E., \& Vega, L. (2014). Oral and written language skills for reading and writing in preschool children. Avances en Psicología Latinoamericana, 32(1), 21-35. doi:http://dx.doi. org/10.12804/apl32.1.2014.02

Guarneros, R. (2013). Análisis del desarrollo de las habilidades lingüisticas orales y escritas en niños preescolares (Tesis de Doctorado). UNAM: Ciudad Universitaria, México.

Hipfner-Boucher, K., Milburn, T., Weitzman, E., Greenberg, J., Pelletier, J., \& Girolametto, L. (2014). Relationships between preschoolers' oral language and phonological awareness. First Language, 34(2), 178-197.

Jason, L.; Jeffrey, M; McDonald; M., \& Francis, J. (2006). Phonological processing and emergent literacy in younger and older preschool. Annals of Dyslexia, 57(2), 113.

Johnson, C., \& Goswami, U. (2010). Phonological awareness, vocabulary, and reading in deaf children with cochlear implants. Journal of speech, language, and hearing research: JSLHR, 53(2), 237-61.

Kim, Y., Petscher, Y., Foorman, B., \& Zhou, Ch. (2010). The Contributions of Phonological Awareness and Letter-Name Knowledge to Letter-Sound Acquisition--A Cross-Classified Multilevel Model Approach. Journal of Educational Psychology, 102(2), 313-326.

Lonigan, C. J., Schatschneider, C., \& Westberg, L. (2008). Identification of children's skills and abilities linked to later outcomes in reading, writing, and spelling. In Developing early literacy: Report of the National Early Literacy Panel (pp. 55-106). Washington, DC: National Institute for Literacy.

McBride-Chag, C. (2014). The development of phonological processing and language for reading. Ed. C. McBride-Chag. Children`s Literacy Development. United States: Routledge.

Menard, S. (1991). Longitudinal research. SAGE University paper: London. Cap 3, pp. 22-31.

Nesselroade, J. \& Baltes, P (1979). Longitudinal research in the study of behavior and development. New York: Academic Press.

Pataki, K. W., Metz, A. E., \& Pakulski, L. (2014). The effect of thematically related play on engagement in storybook reading in children with hearing loss. Journal of Early Childhood Literacy, 14(2), 240-264,357.

Sénéchal, M (2012). Child language and literacy development at home. Ed. B. Wasik Handbook on family literacy. 38-50.

Sénéchal, M. \& LeFevre, J.-A. (2014), Continuity and 
Change in the Home Literacy Environment as Predictors of Growth in Vocabulary and Reading. Child Development, 85: 1552-1568. doi: 10.1111/cdev.12222

Sidman, M. (1978). Tácticas de investigación científica. Barcelona: Fontanella

Silva, A. (2004). Métodos cuantitativos en psicología: un enfoque metodológico. México: Trillas.

Simard, D., Foucambert, D., \& Labelle, M. (2013). Examining the Contribution of Syntactic and Metasyntactic Abilities to Reading Comprehension among Native and Non-Native Speakers. The Metalinguistic Dimension in Instructed Second Language Learning, 45.

Sittner, B., \& Catts, HW. (2011). The use of a dynamic screening of phonological awareness to predict risk for reading disabilities in kindergarten children J Learn Disabil, 44(4).

Skibbe, L. E., Bindman, S. W., Hindman, A. H., Aram, D., \& Morrison, F. J. (2013). Longitudinal Relations Between Parental Writing Support and Preschoolers' Language and Literacy Skills. Reading Research Quarterly, 48(4), 387-401. doi: 10.1002/rrq.55

Susperreguy, M. I., Strasser, K., Lissi, M., R. \& Mendive, S. (2007). Creencias y Prácticas de literacidad en familias chilenas con distintos niveles educativos. Revista Latinoamericana de Psicología, 39(002), 239-251.

Thatcher, K.Wagner, R., Torgesen, J., \& Rashotte, C. (2011). Comparing Two Forms of Dynamic Assessment and Traditional Assessment of Preschool Phonological Awareness. J Learn Disabil, 44, 4, 313-321.

Vance, M., Stackhouse, J., \& Wells, B.(2005). Speechproduction skills in children aged 3-7 years. International Journal of Language \& Communication Disorders. 40(1), 29-48.

Vega, P., L. (2001). Cuestionario de Actividades Alfabetizadoras para padres y cuidadores. Maestría en Psicología. Residencia en Psicología Escolar, Facultad de Psicología, UNAM

Vega, P., L. (2003). Análisis de la contribución de factores involucrados en el desarrollo de la alfabetización en niños preescolares (Tesis de Doctorado de Psicología). UNAM: México

Vega, P., L. (2008). Listas de Cotejo para la Observación de
Oportunidades de Desarrollo de los niños. Maestría en Psicología. Residencia en Psicología Escolar, Facultad de Psicología, UNAM

Vega, P., L., \& Macotela, S. (2006). Alfabetización, retos y perspectivas. México: Facultad de Psicología UNAM.

Vega, P., L., \& Macotela, S. (2008). Desarrollo de la alfabetización en niños preescolares. México: UNAM

Vega, P., L., \& Rangel, A. (2009). Lista de Cotejo de Actividades Alfabetizadoras. Maestría en Psicología. Residencia en Psicología Escolar, Facultad de Psicología, UNAM

Wood C, S., Bowyer S., Jackson, Tarczynski ML-B, Plester B., (2011) Un estudio longitudinal de la mensajería de texto para niños y desarrollo de la alfabetización. British Journal of Psychology,102(3), 431-42. necesidad de permiso expreso de sus autoras con la única condición de que no se puede usar con fines directamente comerciales y los términos legales de cualquier trabajo derivado deben ser los mismos que se expresan en la presente declaración. La única condición es que se cite la fuente con referencia a la Revista Digital Internaciona de Psicología y Ciencia Social y a sus autoras. 


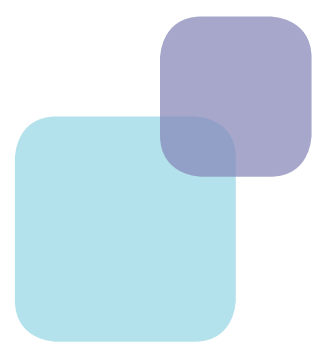

\section{MetA-ANÁlisis Del ARTículo}

A continuación se muestran los análisis de las puntuaciones otorgadas a este artículo por sus revisores 


\section{Dimensión Cuantitativa}

\section{Perfil de Evaluación entre Pares}
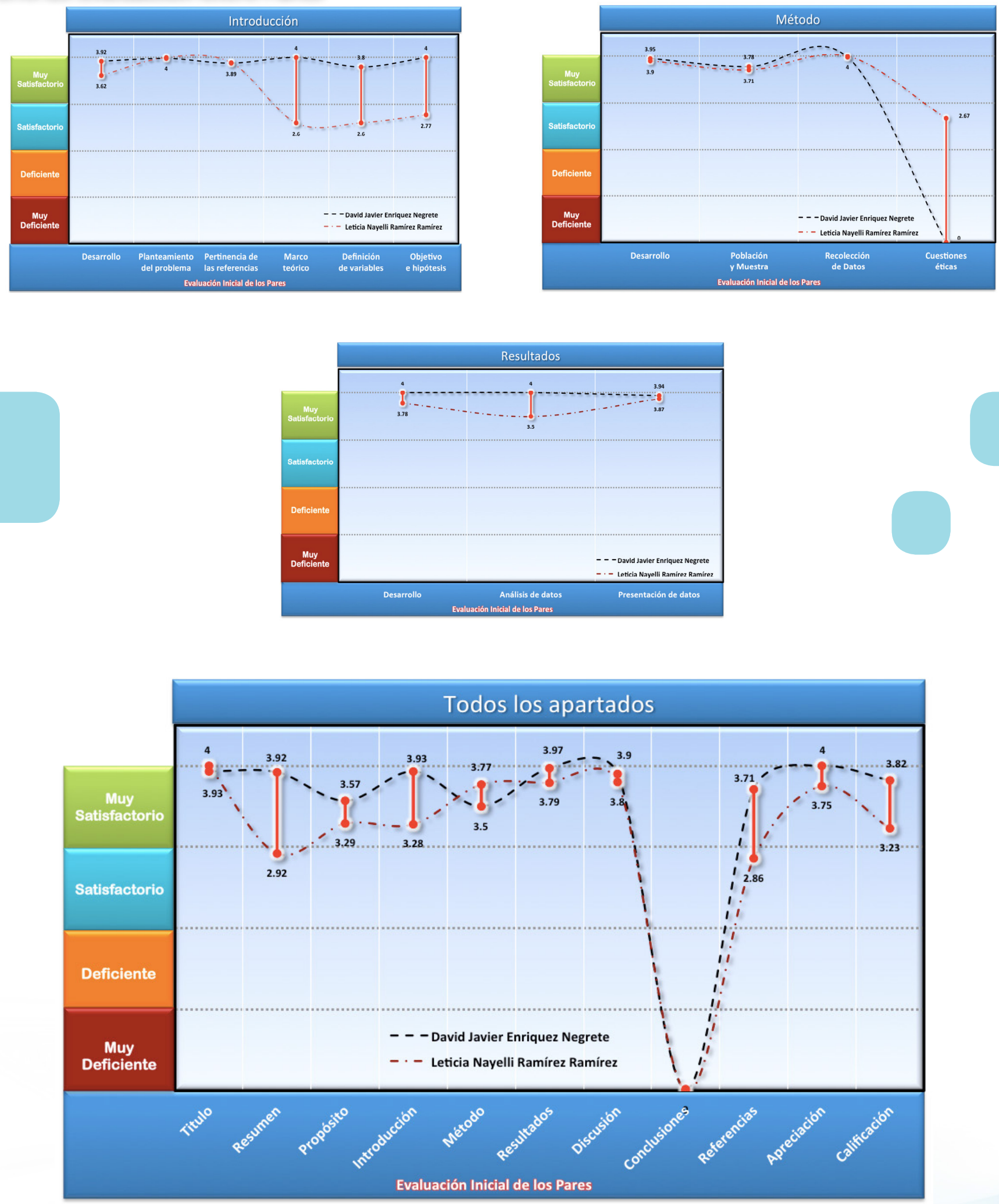

\section{0}




\section{Índice de Concordancia}

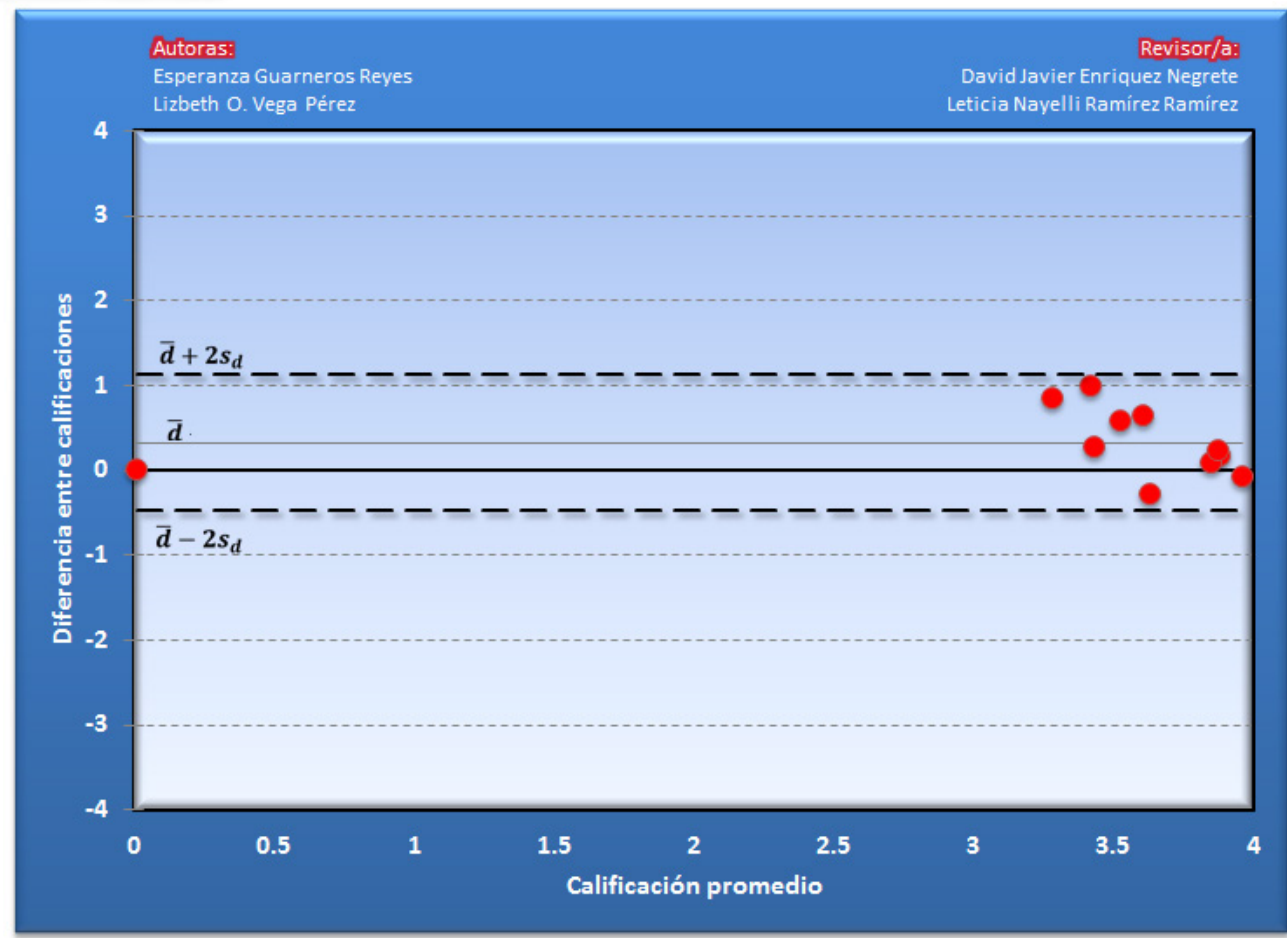

\section{Índice de Acuerdo}

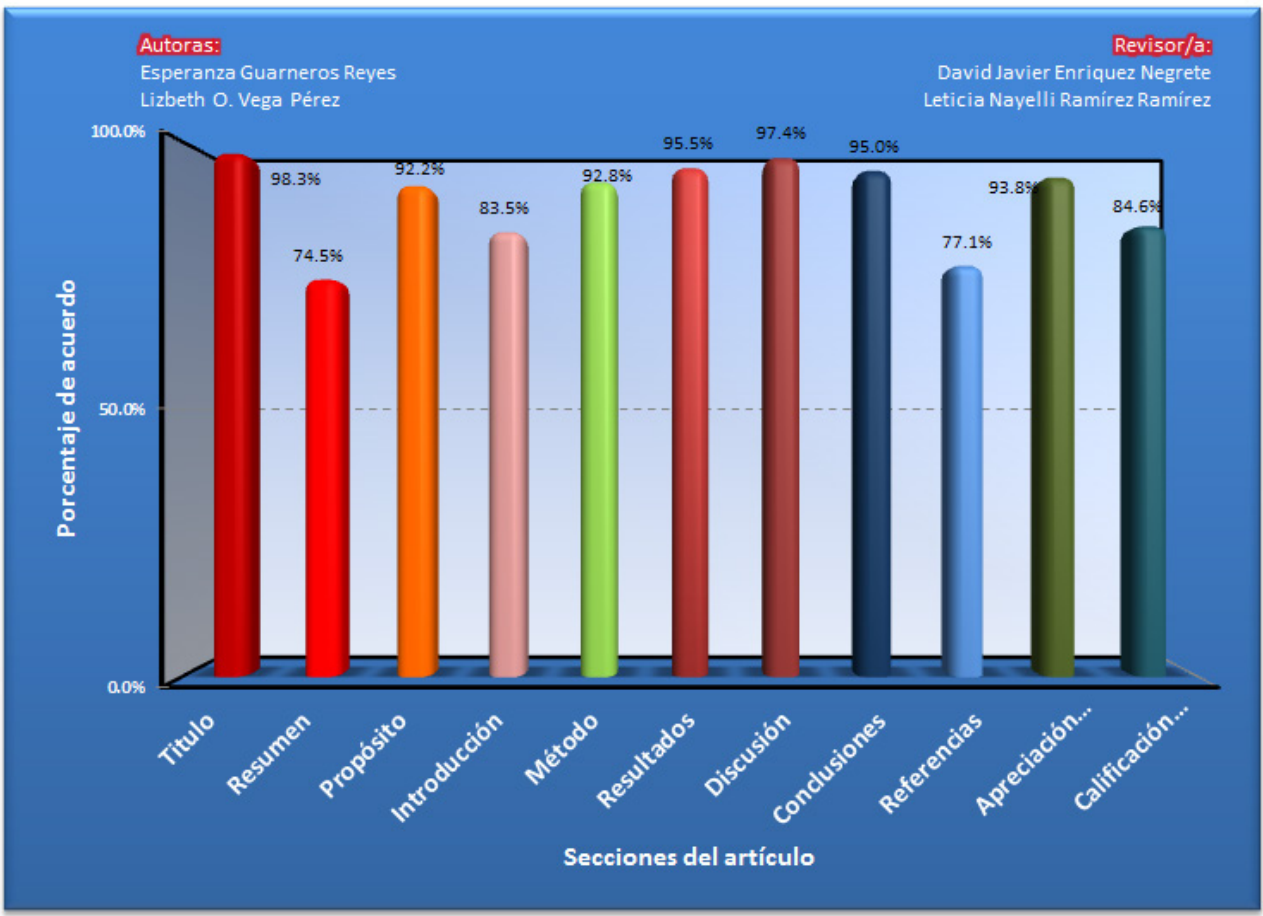




\section{Dimensión Cualitativa}

\begin{tabular}{|c|c|}
\hline ReVISOR 1 & REVISOR 2 \\
\hline Leticia Nayelli Ramírez Ramírez & David Javier Enriquez Negrete \\
\hline \multicolumn{2}{|c|}{ Título/AutoríA } \\
\hline $\begin{array}{l}\text { Comunica el objetivo del estudio y es claro. No requiere } \\
\text { modificaciones. }\end{array}$ & Ninguna. \\
\hline \multicolumn{2}{|c|}{ RESUMEN } \\
\hline $\begin{array}{l}\text { Falta desarrollar varias secciones del resumen. Queda } \\
\text { en claro en la parte de objetivo y resultados. Las } \\
\text { conclusiones no están descritas en el resumen. }\end{array}$ & Ninguna \\
\hline \multicolumn{2}{|c|}{ Próposito del Estudio } \\
\hline $\begin{array}{l}\text { En la sección de Introducción faltaría desarrollar las } \\
\text { hipótesis y las preguntas de investigación. De ahí que, } \\
\text { en las demás secciones (método, resultados, discusión) } \\
\text { no aparece el vínculo directo con las preguntas de } \\
\text { investigación. Es necesario dejar claro este punto. }\end{array}$ & $\begin{array}{l}\text { En el marco teórico no se presenta el propósito del } \\
\text { estudio como hipótesis o pregunta, sino se deriva de } \\
\text { la argumentación y del análisis de estudios empíricos. } \\
\text { Para cumplir con el criterio de la revista solicitado } \\
\text { solamente habría que plantear la pregunta de } \\
\text { investigación la cual se complementaría con el objetivo } \\
\text {-el cual es claro-o como alternativa redactar la hipótesis } \\
\text { sobre el resultado que se espera. }\end{array}$ \\
\hline
\end{tabular}

INTRODUCCIÓN

Se sugiere delinear desde el comienzo de este apartado la relevancia del estudio y la interacción entre variables que se pretenden estudiar. En la revisión de la literatura, se sugiere incluir más referencias actualizadas y nacionales. El marco teórico se menciona escuetamente pero no aparece desarrollado. Igualmente faltaría desarrollar las hipótesis y los objetivos específicos.
Valdría la pena como primer contextualizar la relevancia de estudiar la conciencia fonológica, es decir, ¿qué utilidad tiene estudiar el tema?, ¿para qué puede servir? y bajo este contexto enlazar con el primer párrafo del marco teórico. Esto ayudaría a delinear y describir la relevancia del estudio de una manera más clara.

\section{MÉTOdO}

En el apartado de participantes se sugiere justificar el tamaño de la muestra y qué tan representativa es de la población objetivo. En las consideraciones éticas se sugiere describir cómo se les mencionó a los niños que fueron seleccionados que participarían en el estudio, cómo se aseguró el anonimato y la confidencialidad de los participantes, así como, los riesgos potenciales del estudio. Se sugiere anexar la constancia de consentimiento informado que se les hizo llegar a los padres.
Se especifica que los padres son de clase media-baja, que características implica ser de la clase media-baja, esto está determinado por el nivel de ingreso mensual? Por las condiciones estructurales de la vivienda? O como se determinó la clase social? Habría que desarrollar un poco más la sección donde se especifica que se les notificó a los padres con una circular sobre el estudio, pero en qué términos, se firmó de conformidad de que sus hijos participarán, que decía la circular, se especificó que era voluntario, que esto no tendría repercusión en las calificaciones de sus hijos, que los datos se utilizarán exclusivamente con fines estadísticos, se solicitaron datos personales o quedo en anonimato, etc. Sería importante describir un poco estos detalles. 


\section{RESULTADOS}

Se sugiere que en la sección de resultados se establezca una correlación con los objetivos e hipótesis de investigación.

Ninguno.

\section{Discusión}

Se sugiere desarrollar la parte de las limitaciones del estudio. Y ampliar el párrafo en el que se habla de las futuras investigaciones que se derivan del estudio.

\begin{tabular}{l|l} 
No aparece en el texto la sección de Conclusiones. & Ninguno.
\end{tabular}

\section{ReFERENCIAS}

Revisar el listado de referencias, la mayoría aparece sin formato APA, y una de ellas no está citada en el texto.
Si el criterio de la revista es de al menos el 50\% de referencias de los últimos cinco años, no se cumple con el criterio, sin embargo, la mayor parte de las referencias son del 2000 a la fecha, para ajustarse al criterio solicitado por la revista valdría la pena actualizar algunas referencias.

\section{Impresión General del Manuscrito}

En general el trabajo presenta un nuevo abordaje del desarrollo de la consciencia fonológica y presenta datos empíricos sustentados. Sin embargo, faltaría desarrollar con más claridad las hipótesis y pregunta de investigación. Además de corregir cada detalle que se describió de las secciones. Revisar la puntuación y los errores de digitación. Así como el espaciado entre palabras y el formato del texto.
Se adjuntó en el texto algunas observaciones para corregir algunos errores de digitación. Revisar archivo adjunto.

\section{COMENTARIO POR DICTAMEN}

La contribución es suficientemente sustancial, tiene fundamentos empíricos y lógicos. Sin embargo, considero que es necesario hacer una revisión profunda de los detalles señalados en cada sección. Desarrollar la fundamentación del estudio (preguntas de investigación, hipótesis), ahondar en la discusión, incluir la sección faltante de Conclusiones y modificar el listado de referencias. Al realizar lo anterior, el manuscrito cumplirá con los estándares de calidad que solicita la Revista Digital Internacional de Psicología y Ciencia Social.
En la actualidad existen dos grandes políticas editoriales Porque es un estudio de relevancia para la psicología en el área de desarrollo de lenguaje, aporta datos en torno a una población poco estudiada respecto a la consciencia fonológica -preescolares-, existe una aportación más allá de los tipos estudios de análisis lineales, lo cual implica brindar una aportación teórica y metodológica. 


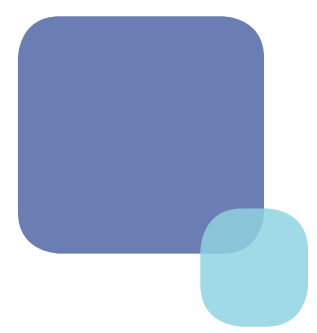

\section{IR A LA Historia del Proceso Editorial}


